\title{
Editorial: A Timely Contribution to a Half-Century-Old Topic
}

Isn't the topic this paper addresses - thermodynamic properties of elemental solids-more than half-a-century old? What fundamental new insights is the paper providing? These were some of the main questions we had when we received the submission by Glensk et al. of their paper entitled, "Breakdown of the Arrhenius Law in Describing Vacancy Formation Energies: The Importance of Local Anharmonicity Revealed by Ab initio Thermodynamics" [Phys. Rev. X 4, 011018 (2014)]. While we recognized the technical quality of the paper, we had our reservations about its currentness, its novelty, and its significance. The expert input we received through the review process, however, persuaded us that there was a strong case for publishing it in PRX. There is currently a concerted international effort to assess critically thermodynamic data on elemental solids. This paper, which breathes fresh and revitalizing air into the "classical" topical area, is a timely, high-quality, and important contribution and deserves broad dissemination, in particular, to the large number of experimentalists and theorists who do both basic and applied materials physics research.

We are very happy that Göran Grimvall from the Royal Institute of Technology (KTH), Stockholm, Sweden, has agreed to introduce the publication of this paper with his brief Commentary on why the paper is an important contribution. We hope that you will enjoy both the Commentary and the paper itself.

The Editors

\section{New Light on the Arrhenius Law for Thermal Defects in Solids}

Göran Grimvall, Royal Institute of Technology (KTH), AlbaNova University Center, SE-106 91 Stockholm, Sweden

A Commentary on:

Breakdown of the Arrhenius Law in Describing Vacancy Formation Energies: The Importance of Local Anharmonicity Revealed by Ab initio Thermodynamics

A. Glensk, B. Grabowski, T. Hickel, J. Neugebauer

Phys. Rev. X 4, 011018 (2014)

Defects of various kinds govern much of the behavior of solids. While energies of bulk crystalline solids are now obtained with an impressive accuracy by use of $a b$ initio methods, calculating the energy associated with the formation of a lattice defect ("defect formation energy" in short) is still a challenge because the local electron density, a key parameter in the $a b$ initio work, varies rapidly near a defect. The half-century-old experimental tradition for obtaining the formation energy of thermally created defects relies on the use of an Arrhenius law relating the defect concentration $c$ to temperature $T$ : experimental data for $\ln c$ is plotted versus $1 / T$ and the defect formation energy is obtained from the slope of the plot. On the other hand, current theoretical calculations of $E_{f}$ are performed at $T=0 \mathrm{~K}$ in a static lattice containing the defect.

There are two fundamental problems bridging these two approaches: First, the Arrhenius law is only an approximate model; second, the experimental data cover a rather narrow temperature interval just below the solid's melting temperature whereas the theoretical value corresponds to zero temperature. The paper by Glensk et al. [Phys. Rev. X 4, 011018 (2014)] has clarified the long-outstanding situation in depth. Using state-of-the-art theoretical ab initio approaches, they are able to reach a number of substantive results and new insights, in particular, the quantitative demonstration that anharmonic vibrational effects cannot be neglected and the realization that the assumption central to the empirically applied Arrhenius law-a temperature-independent formation entropy - must be replaced by an entropy that varies with temperature. The paper also represents an important step for future theoretical work on the modeling of materials in practical use.

Published by the American Physical Society under the terms of the Creative Commons Attribution 3.0 License. Further distribution of this work must maintain attribution to the author(s) and the published article's title, journal citation, and DOI. 
About the Commentary author:

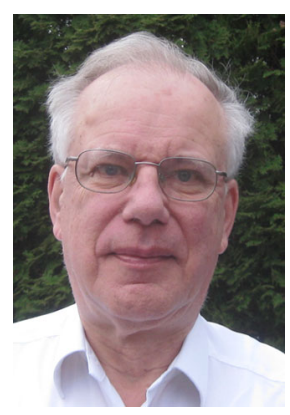

Göran Grimvall is professor emeritus in the theory of materials at the Royal Institute of Technology (KTH), Stockholm, Sweden. His research interest is thermophysical properties of materials.

Published 10 February 2014

DOI: 10.1103/PhysRevX.4.010001 